\title{
On the growth of Iberian firms: an empirical analysis
}

\author{
R. Pascoal ${ }^{\mathrm{a}}$, M. Augusto ${ }^{\mathrm{a}}, \mathrm{H}$. Rocha $\mathrm{a}^{\mathrm{a}, \mathrm{b}, *}$ \\ ${ }^{a}$ CeBER \& FEUC, Av. Dias da Silva 165, 3004-512 Coimbra, Portugal. \\ ${ }^{b}$ INESC-Coimbra, Rua Silvio Lima, Polo II, 3030-290 Coimbra, Portugal.
}

\begin{abstract}
The growth dynamics of firms have been object of numerous studies by econophysicists. The vast majority of these studies was done for large developed countries including the United States of America (US), France or Italy. These studies were conducted for countries with strong economies and whose business fabric has different characteristics from countries such as Portugal or Spain, with more large-sized firms and less micro-sized firms. It is thus important to study the growth dynamics of firms in Portugal and Spain, countries with economies that typically display anemic growth indexes and have a larger percentage of micro-sized firms. Considering two large samples of Portuguese and Spanish firms, we find that size distribution of firms is well fitted by the generalized Pareto distribution. Moreover, the distribution of Iberian firms annual growth rates is best approximated by the Cauchy distribution rather than the Laplace distribution. Firms growth standard deviation conditional on size is well approximated by power-law relationships with a scaling exponent close to $\frac{1}{2}$ for micro/small-sized firms and close to 0 for medium/large-sized firms.
\end{abstract}

Keywords: Iberian firms, Firm growth, Power-law distributions, Econophysics

\section{Introduction}

The characterization of the distribution of firm growth is intimately related to the assumptions underlying the size distribution itself, and it concerns not only the kind of distribution of growth rates but also its mean and standard deviation conditional on size. A review of theoretical analysis and stylized facts found in literature of the field is in order. Let $S_{t}$ denote firm size at time $t$. Firm growth rate, $r$, is defined as

$$
r_{t+1}=\ln \left(\frac{S_{t+1}}{S_{t}}\right) .
$$

\footnotetext{
${ }^{*}$ Corresponding author. Tel.: +351 239 790527; fax: +351 239403511.

Email addresses: ruiapsp@fe.uc.pt (R. Pascoal), maugusto@fe.uc.pt (M. Augusto), hrocha@mat.uc.pt (H. Rocha)
} 
The seminal papers concerning firm growth rate distribution are Stanley et al. [1] and Amaral et al. [2]. It was found that the distribution that better fits US manufacturing firms is the exponential Laplace where the conditional density is given by

$$
p\left(r_{t+1} \mid s_{t}\right)=\frac{1}{\sqrt{2} \sigma\left(s_{t}\right)} \exp \left(-\frac{\sqrt{2}\left|r_{t}-\bar{r}\left(s_{t}\right)\right|}{\sigma\left(s_{t}\right)}\right),
$$

where $s_{t}=\ln \left(S_{t}\right)$. Remark that $\bar{r}\left(s_{t}\right)$ and $\sigma\left(s_{t}\right)$ are the unknown parameters which are estimated by fitting the data to this distribution and represent the mean and the standard deviation of growth rates, respectively. To improve the quality of the estimators, we can use the time average of data when several years are available for each firm. The distribution has been confirmed for fitting many other samples (e.g., $[3,4,5,6]$; these last two consider also a generalization, the Subbotin distribution). Exponential Laplace places far more weight on the tails then does the Normal distribution that would result from the classic Gibrat model [7] where a multiplicative process for size and growth rate is assumed to be independent of the corresponding initial size. Exponential Laplace can be obtained through a model with regression towards the mean. On the other hand, other distributions also allows suitably to adjust heavy tails (e.g., Xie et al. [8] fit Student distribution). The use of distributions that combine a Laplace central region with power-law tails has also been proposed, see, e.g., Fu et al. [9] or Buldyrev et al. [10], to improve the tails' fitting of the Laplace distribution. However, Williams et al. [11] show that such ad hoc adjustments are not necessary. Using a richer database and testing more theoretical distributions, Williams et al. [11] found that the distribution of firm growth rates (as well as economic profit rates [12] and GDP growth rates [13]) are best approximated by the Cauchy distribution whose conditional density is given by

$$
p\left(r_{t+1} \mid s_{t}\right)=\frac{1}{\pi \sigma\left(s_{t}\right)}\left(\frac{\sigma^{2}\left(s_{t}\right)}{\left(r_{t}-\mu\left(s_{t}\right)\right)^{2}+\sigma^{2}\left(s_{t}\right)}\right),
$$

where $\mu$ is the location parameter and $\sigma$ is the scale parameter. The Cauchy distribution is a heavy-tailed distribution whose variance has an important economic feature - both very low and very high firm growth rates occur much more often than predicted by the Gaussian distribution [11].

By decomposing the observations in terms of classes of size, Stanley et al. [1] and Amaral et al. [2] also point out the existence of a scaling relation between $\sigma\left(s_{t}\right)$ and $S_{t}$, represented by

$$
\sigma\left(s_{t}\right) \sim S_{t}^{-\beta}
$$

where the scaling exponent is negative (i.e., $\beta>0$ ). That is, the standard deviation of size growth rate decreases, in mean, with increasing firm size. In fact, Hymer and Pashigian [15] already noted this negative relation and explained this behavior in the context of firms divided on units of equal size and independent which would lead to a coefficient $\beta$ equal to $\frac{1}{2}$. Amaral et al. [2] also 
justifies this fact in the context of multi-divisional firms by noting that larger firms tend to be more diversified than smaller ones. Diversification is in general associated with reduction of risk since a negative result in some units of the firm is likely to be (more or less) compensated by positive returns in others, unless their results are perfectly correlated. Nonetheless, the fact that the divisions of the firms are linked and not working independently explains why in empirical studies $\beta<\frac{1}{2}$. The value of $\beta$ is inversely correlated with dependence between units of the firm. The extreme value $\left(\beta=\frac{1}{2}\right)$ is attained when units are independent (due to the law of Large Numbers). Even in the case of independence, $\beta$ may be inferior to $\frac{1}{2}$ if the number of firm units increases with size, which is likely to happen. Bottazzi and Secchi [14] analyze relation (3) by modeling the numbers of active sub-markets of the firm as a point process where intensity function depends on size, and, on the more radical version, on the process itself (it is a self-exciting process). In this last case, the usually observed relation (3) can be explained without refuting the law of Large Numbers. An aspect even more directly related to Gibrat law is the relation between mean growth and size: there should be no such relation, given the assumption of growth independence on size. Nonetheless, in empirical studies this result is only confirmed in the case of large firms. In fact, for small firms, a negative relation is found: smaller firms tend to grow faster, resulting in mean reversion for size. The seminal study in this direction is the one of Mansfield [16] who distinguishes the behavior of firms according to the distinction between surviving and exiting firms and according to size. See also Coad [17] and references therein for the discussion of these topics.

The main purpose of this paper is to study and characterize the distribution of firm growth in two large samples of Portuguese and Spanish firms. It is important to study the growth dynamics of firms in Portugal and Spain, countries with distinctive economic and business features from large developed countries typically considered for the vast majority of studies found in the literature. Some of the distinctive characteristics of Portugal and Spain include an even larger percentage of micro-sized firms and a pathologically economic anemic growth. The paper is organized as follows. In the next section a brief data description is made. An overview of the Portuguese and Spanish firms is provided in Section 3. Section 4 presents the analysis and the results. In the last section we have the discussion and conclusion.

\section{Data description}

This study is implemented on two sets of Portuguese and Spanish firms on the period from 2011 to 2016. The main source of information was the IBAS (Iberian Balances Analysis System), provided by Bureau Van Dijk (property of Moodys Analytics), containing accounting and financial information of Portuguese and Spanish firms. We used the $\mathrm{NACE}^{1}$ classification of economic

\footnotetext{
${ }^{1}$ Nomenclature statistique des activits conomiques dans la Communaut europenne
} 
Table 1: Size structure of existing Portuguese and Spanish firms in at least 2 years in the period 2011 to 2016.

\begin{tabular}{|c|c|c|c|c|}
\hline & \multicolumn{2}{|c|}{ Portugal } & \multicolumn{2}{|c|}{ Spain } \\
\hline & \# & $\%$ & \# & $\%$ \\
\hline All firms & 137232 & 100,00 & 475446 & 100,00 \\
\hline micro-sized & 102557 & 74,73 & 352183 & 74,07 \\
\hline small-sized & 29009 & 21,14 & 104712 & 22,02 \\
\hline medium-sized & 4867 & 3,55 & 15530 & 3,27 \\
\hline large-sized & 799 & 0,58 & 3021 & 0,64 \\
\hline
\end{tabular}

activities (Rev. 3), built in the European Union. The disaggregation of activities into sectors and subsectors is described by associating to each (sub)sector a code. In each code, an additional level of disaggregation involves adding a new digit.

The samples are selected from IBAS database so that: (i) only limited liability and public limited companies are considered, (ii) excluding those in the Financial and insurance activities (NACE codes from 64 to 66), and Public administration and defense and compulsory social security (NACE code 84), (iii) only those that existed and are in activity (volume of Sales and Total Assets both greater than 1000 euros) in the period are accounted for, (iv) only those including information on the number of subsidiaries are retained. As a result of these criteria, final samples of 137,232 Portuguese firms and 475,446 of Spanish firms are obtained.

Micro, small and medium-sized firms (SMEs) are defined, according to the EU recommendation 2003/361, as follows. Medium firm will have a staff headcount of at least 50 up to less than 250 workers, a turnover of more than 10 up to at most 50 million euros or a total balance sheet of more than 10 up to at most 43 million euros. Small firm will have a staff headcount of at least 10 up to less than 50 workers, a turnover of more than 2 up to at most 10 million euros or a total balance sheet of more than 2 up to at most 10 million euros. Micro firm will have a staff headcount of less than 10 workers, a turnover of at most 2 million euros or a total balance sheet of at most 2 million euros. Based on these definitions, we have the size structure for Portuguese and Spanish firms displayed in Table 1.

\section{Overview of the Portuguese and Spanish firms}

Before presenting the main results of this research, it is worthwhile to briefly describe some distinctive features of the Portuguese and Spanish business fabric and its evolution in recent years. An exhaustive description of the overall behavior of the Portuguese and Spanish economy is out of the scope of this paper, 


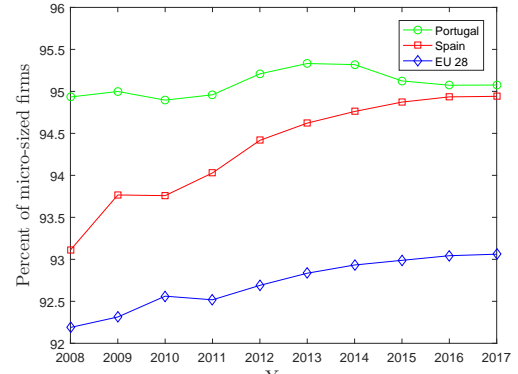

(a)

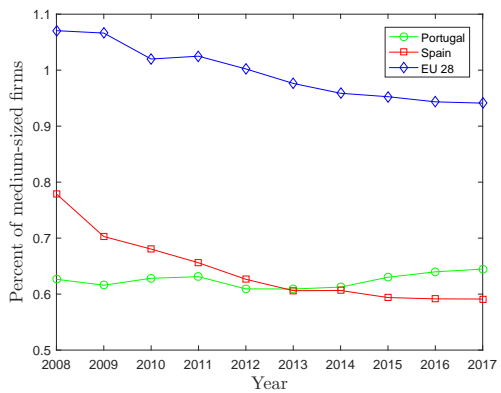

(c)

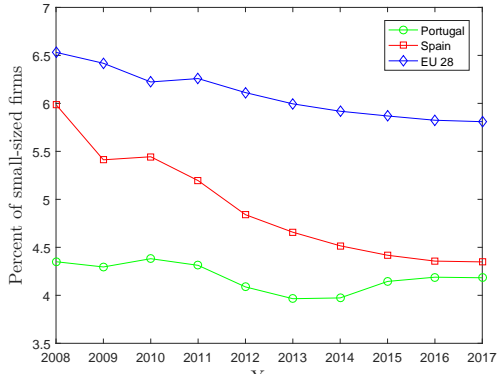

(b)

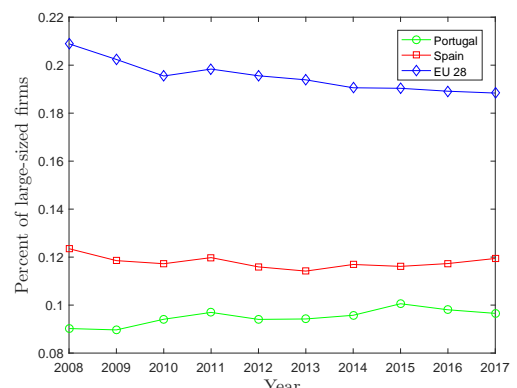

(d)

Figure 1: Percent of micro (1(a)), small (1(b)), medium (1(c)) and large-sized (1(d)) firms in Portugal, Spain and European Union (EU 28). Source: Annual Report on European SMEs [18].

but the specific characteristics of the Portuguese and Spanish firms discussed next may help to interpret the results obtained in this study.

One of the main features of Portuguese and Spanish firms is that SMEs are the core of Iberic economy, representing more than $99 \%$ of companies in the non-financial sector and more than $80 \%$ of the persons employed [18]. Although the weight of SMEs in the European Union (EU 28) is similar, one distinctive characteristic of Portugal and Spain is a smaller ratio of large-sized firms and a larger proportion of micro-sized firms as observed in Fig. 1. In the past ten years, the proportion of large-sized firms in Iberia is half of the proportion in EU 28 while micro-sized firms presented a larger weight. Furthermore, while the weight of large-sized firms is stable in the past decade, the proportion of micro-sized firms shows an increasing trend for Iberian firms.

Another distinctive characteristic of Portugal and Spain is its economic anemic growth. The annual percentage growth rate of GDP per capita based on constant local currency is displayed in Fig. 2 for Portugal, Spain, and EU 28. The mean GDP per capita growth for the last decade was $-0.1 \%, 0.3 \%$ and $0.8 \%$ for Portugal, Spain and EU 28, respectively. The worst economic performance of Iberian countries as compared to EU 28 is even more accentuated 


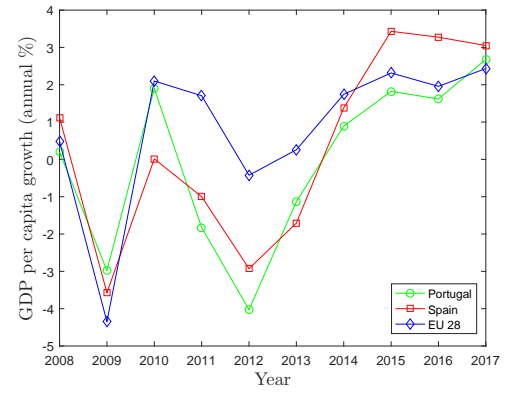

Figure 2: Annual percentage growth rate of GDP per capita based on constant local currency. Source: World Bank national accounts data [19].

when compared with the US that had $1.4 \%$ of mean GDP per capita growth in the last decade and presented a more differentiated business fabric with a larger proportion of medium and large-sized firms [19].

\section{Analysis and Results}

This section presents the main statistical findings of Portuguese and Spanish firm dynamics. We divide our results into four main axes. We begin by exploring the size distribution, paying particular attention to the micro and small-sized firms where more data exists. Next, we study the distribution of growth rates, followed by the analysis of the size-dependent mean growth and standard deviation. Finally, for different sizes that emerged in the third axis studied, the distribution of growth rates is analyzed all over again.

\subsection{Size distribution}

Firms' total sales was used as measure of size, $S_{t}$, at time $t$. For one of the middle years in this study, 2014, Fig. 3 displays the $\log _{10}$ of $S_{t}$ on the horizontal axis and the $\log _{10}$ of the frequency on the vertical axis. For each of the remaining years of this study, the resulting plots are similar. We find that the raw data is well fitted by the generalized Pareto distribution. The probability density function of the generalized Pareto distribution with threshold parameter $\mu$, scale parameter $\sigma$, and shape parameter $k \neq 0$ is

$$
p(x \mid k, \sigma, \mu)=\frac{1}{\sigma}\left(1+k \frac{x-\mu}{\sigma}\right)^{-1-\frac{1}{k}},
$$

for $x>\mu$, when $k>0$, or for $\mu<x<\frac{\sigma}{k}$, when $k<0$. Note that if $k>0$ and $\mu=\frac{\sigma}{k}$ then the generalized Pareto distribution corresponds to the Pareto distribution. In previous works, it has been reported that the Pareto distribution fits well the size distribution of firms (see, e.g., Axtell et al. [5] or Pascoal et al. [20]). In particular, the Pareto distribution has been preferred to the 


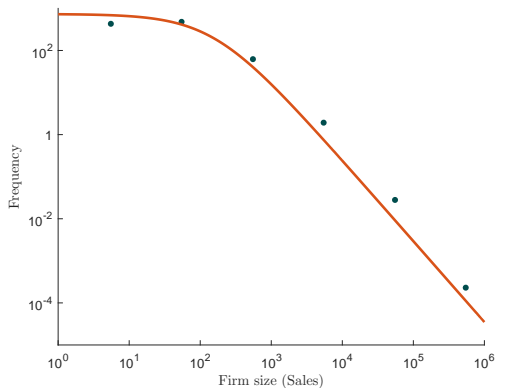

(a)

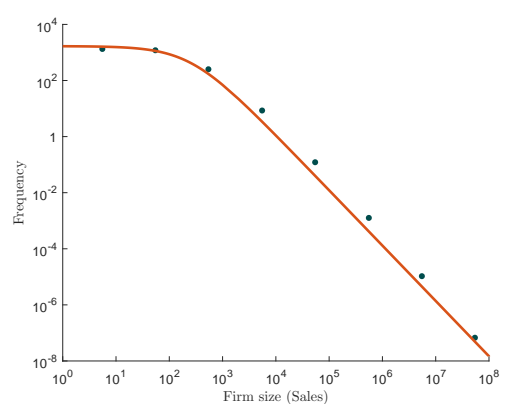

(b)

Figure 3: Size distribution of the Portuguese firms (Fig. 3(a)) and the Spanish firms (Fig. $3(\mathrm{~b})$ ) in log-log scale for the year 2014. The raw data is well fitted by the generalized Pareto distribution with parameters $k=1.083(1.071,1.093), \sigma=169.1(167.2,170.9)$ and $\mu=0$ for the Portuguese firms and parameters $k=1.028(1.022,1.034), \sigma=255.6(254.1,257.2)$ and $\mu=0$ for the Spanish firms.

lognormal distribution, considered to be a good fit since the original work of Gibrat [7], mainly because of its superior performance in describing the upper tail of the empirical size distribution [21]. However, the Pareto distribution is a good fit for data that lay around a straight line in the log-log plot, which is not the case for smaller firm sizes (as wasn't exactly the case in Axtell et al. [5]). As a large proportion of the firms are micro/small-sized, it is important to obtain a good fitting for these firm sizes. Thus, for our data, the generalized Pareto distribution is a better fitting for the size distribution of firms than the Pareto distribution.

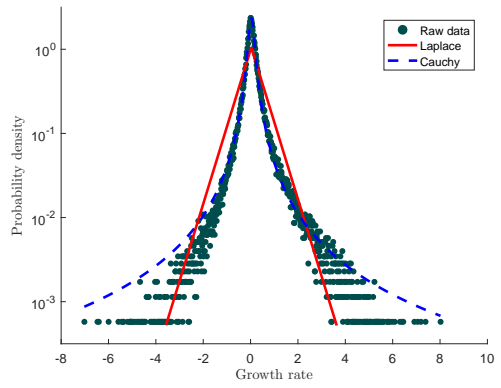

(a)

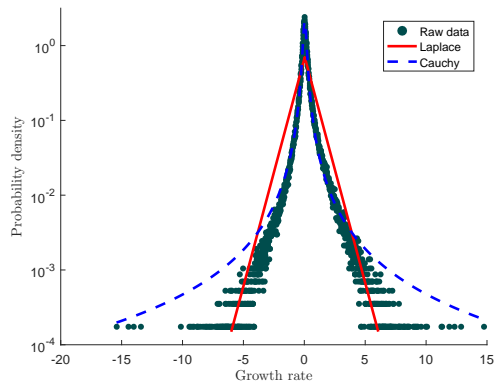

(b)

Figure 4: Empirical probability density functions of growth rates for the Portuguese firms (Fig. 4(a)) and the Spanish firms (Fig. 4(b)). The solid lines correspond to Laplace distributions obtained by fitting the data using Eq. (1) with parameters $\bar{r}\left(s_{t}\right)=0.03$ and $\sigma\left(s_{t}\right)=0.67$ for the Portuguese firms and parameters $\bar{r}\left(s_{t}\right)=0.04$ and $\sigma\left(s_{t}\right)=1.01$ for the Spanish firms. The dashed lines correspond to Cauchy distributions obtained by fitting the data using Eq. (2) with parameters $\mu\left(s_{t}\right)=0.03$ and $\sigma\left(s_{t}\right)=0.14$ for the Portuguese firms and parameters $\mu\left(s_{t}\right)=0.04$ and $\sigma\left(s_{t}\right)=0.15$ for the Spanish firms. 


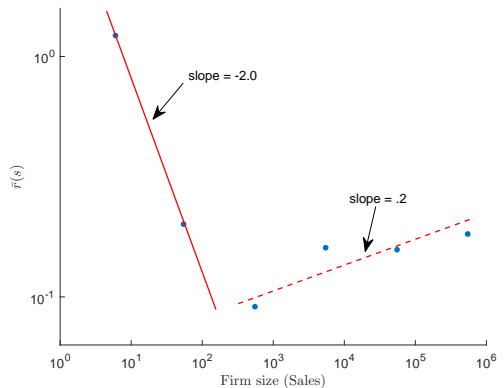

(a)

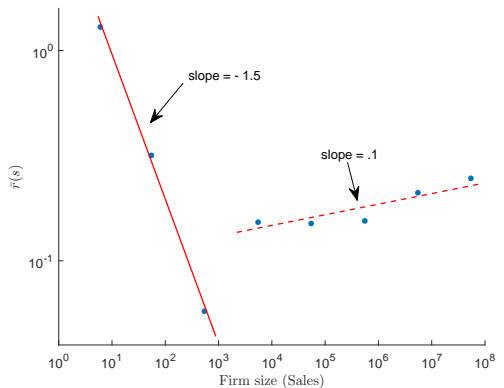

(b)

Figure 5: Mean growth rates of the one-year sales (circles) as a function of the initial firm size values (sales) for Portuguese firms (Fig. 5(a)) and for Spanish firms (Fig. 5(b)).

\subsection{Distribution of growth rates}

The empirical probability density functions of growth rates for Portuguese and Spanish firms are displayed in Fig. 4. We find that the Cauchy distributions obtained by fitting the data using Eq. (2) for both the Portuguese firms and the Spanish firms fit the data better than the Laplace distributions obtained by fitting the data using Eq. (1). These results are congruent with Williams et al. [11] findings that claim that the distribution of firm growth rates is most of the times better fitted by the Cauchy distribution than by the Laplace distribution. The 'stylized fact' of the Laplace distribution being repeatedly considered the most suitable fit for firm growth rates in many studies (see, e.g., $[22,23,24,25,26,27,28,29])$ since the path-breaking study of Stanley et al. [1], have been questioned by other empirical findings. For example, Bottazi et al. [30] show that the growth rates of French firms are even fatter tailed than predicted by Laplace distribution.

\subsection{Size-dependent mean growth and standard deviation}

The relationship between the mean growth rate, $\bar{r}\left(s_{t}\right)$, and the initial firm size values as well as the relationship between the standard deviation of growth rate, $\sigma\left(s_{t}\right)$, and the initial firm size used to calculate the growth rate are now analyzed. In order to study these relationships, data points were sorted in ascending order by their size and grouped in classes of the form $\left(10^{i} ; 10^{i+1}\right)$, $i=0,1,2, \ldots$ For each interval, the mean growth rate, $\bar{r}\left(s_{t}\right)$, and the standard deviation of growth rate, $\sigma\left(s_{t}\right)$, were calculated. Fig. 5 displays the mean growth rates of the one-year sales (2014) as a function of the initial firm size values (sales) for Portuguese firms and for Spanish firms. Fig. 6 displays the standard deviation of the one-year growth rates of the sales (2014) as a function of the initial firm size values (sales) for Portuguese firms and for Spanish firms. It is worth to highlight that these relationships remain remarkably stable for the remaining years of this study. 


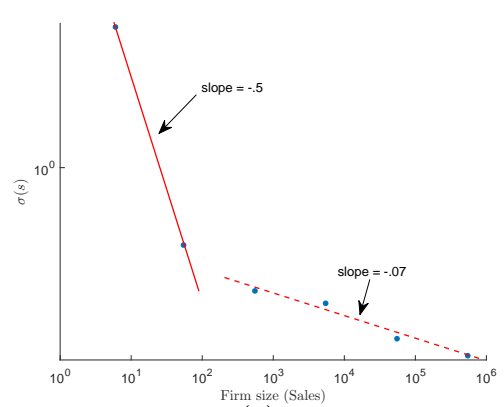

(a)

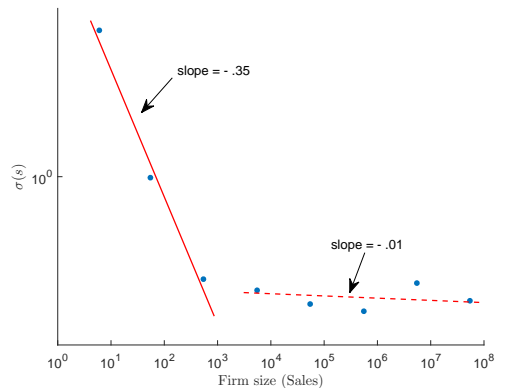

(b)

Figure 6: Standard deviation of the one-year growth rates of the sales (circles) as a function of the initial firm size values (sales) for Portuguese firms (Fig. 6(a)) and for Spanish firms (Fig. 6(b)).

Fig. 5 shows that the mean growth has a linear decay for the first two orders of size magnitude (up to $10^{2}$ ) for Portuguese firms and for the first three orders of size magnitude (up to $10^{3}$ ) for Spanish firms followed by a slight increasing linear trend for the following orders of size magnitude. Despite these trends, note that the mean growth for the first orders of size magnitude present larger values than mean growth for higher orders of size magnitude.

The existence of a scaling relation between the standard deviation of growth rate and the initial firm size values given by Eq. 3 is confirmed in Fig. 6. However, instead of a single scaling relation, we observe two scaling relations: one for the first orders of size magnitude (up to $10^{2}$ for Portuguese firms and up to $10^{3}$ for Spanish firms) with a larger $\beta$ (equal or close to $\frac{1}{2}$ for Portugal and Spain, respectively) and another for the following orders of size magnitude with a smaller $\beta$ (close to 0 for both Portugal and Spain).

\subsection{Distribution of growth rates by size}

The empirical probability density functions for Portuguese and Spanish firms are further analyzed for the size classes that emerged in the previous section. Fig. 7 shows that, despite the Laplace distribution capture fairly well the 'tentshape' of the data, the Cauchy distributions continue to obtain better fittings both for lower orders of magnitude (up to $10^{2}$ for Portugal and up to $10^{3}$ for Spain) and for higher orders of magnitude (greater than $10^{2}$ for Portugal and greater than $10^{3}$ for Spain).

\section{Discussion and conclusion}

In this study, the growth dynamics of Iberian firms were analyzed considering two large samples of Portuguese and Spanish firms. The 'stylized fact' present in literature that the growth rate distribution is 'tent-shaped' was found yet again in this empirical study. This 'stylized fact' means that the frequency of extreme 


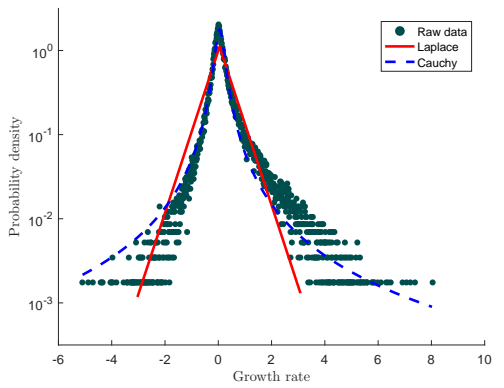

(a)

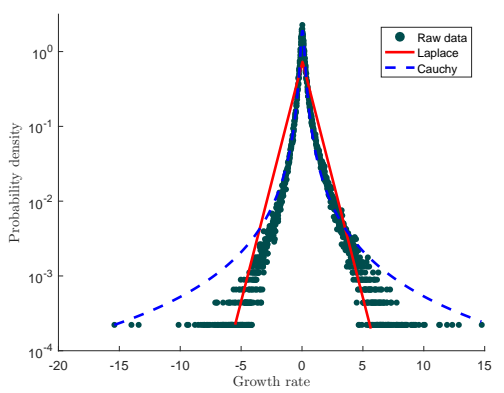

(c)

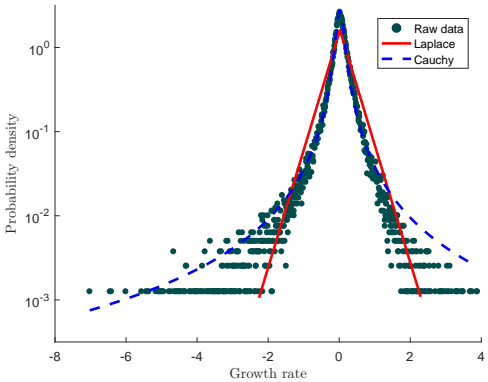

(b)

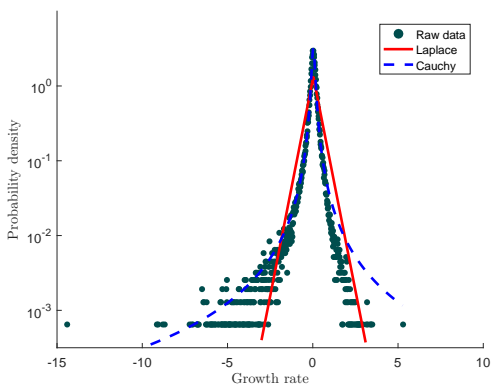

(d)

Figure 7: Empirical probability density functions, $p\left(r_{t+1} \mid s_{t}\right)$, for the micro/small-sized $\left(S_{t} \leq 10^{2}\right)$ Portuguese firms (Fig. 7(a)), for the medium/large sized $\left(S_{t}>10^{2}\right)$ Portuguese firms (Fig. $7(\mathrm{~b})$ ), for the micro/small-sized $\left(S_{t} \leq 10^{3}\right)$ Spanish firms (Fig. $7(\mathrm{c})$ ), and for the medium/large-sized $\left(S_{t}>10^{3}\right)$ Spanish firms (Fig. $7(\mathrm{~d})$ ). The solid lines correspond to Laplace distributions obtained by fitting the data using Eq. (1) with parameters $\bar{r}\left(s_{t}\right)=0.05$ and $\sigma\left(s_{t}\right)=0.64$ in Fig. $7(\mathrm{a}), \bar{r}\left(s_{t}\right)=0.02$ and $\sigma\left(s_{t}\right)=0.44$ in Fig. $7(\mathrm{~b}), \bar{r}\left(s_{t}\right)=0.04$ and $\sigma\left(s_{t}\right)=0.97$ in Fig. $7(\mathrm{c})$, and $\bar{r}\left(s_{t}\right)=0.03$ and $\sigma\left(s_{t}\right)=0.53$ in Fig. $7(\mathrm{~d})$. The dashed lines correspond to Cauchy distributions obtained by fitting the data using Eq. (2) with parameters $\mu\left(s_{t}\right)=0.05$ and $\sigma\left(s_{t}\right)=0.18$ in Fig. $7(\mathrm{a}), \mu\left(s_{t}\right)=0.02$ and $\sigma\left(s_{t}\right)=0.12$ in Fig. $7(\mathrm{~b})$, $\mu\left(s_{t}\right)=0.04$ and $\sigma\left(s_{t}\right)=0.17$ in Fig. $7(\mathrm{c})$, and $\mu\left(s_{t}\right)=0.03$ and $\sigma\left(s_{t}\right)=0.11$ in Fig. $7(\mathrm{~d})$.

growth events is not very low and such extreme growth events contribute significantly for firms' evolution. Despite the Laplace distribution explain reasonably well the growth rate distributions in this empirical study, the Cauchy distribution clearly presents a better fitting to the data, corroborating the findings of Williams et al. [11].

Several studies, considering mostly large firms, indicate a trend for larger firms to have a higher rate of growth (see, e.g., [31, 32, 33]). However, other studies considering larger databases found that, in average, small firms grow faster than large firms (see, e.g., $[34,35,36]$ ). We find that, for our data, Gibrat's law holds for firms above a certain size threshold $\left(10^{2}\right.$ for Portugal and $10^{3}$ for Spain) while for smaller size firms we observe the so-called 'reversion to the mean size'. Although this result is not pioneer (see, e.g., Caves [37]) 
it reinforces the body of research with the empirical notion that smaller-sized firms behavior differs from larger-sized firms. This difference is also observed for the standard deviation of the size-dependent growth. While the scaling relation between the standard deviation of growth rate and size is always negative, the linear relation is evident for the same classes identified for mean growth, with a steepest decrease for the smaller-sized firms. The discussion in Lee et al. [4] presents a good explanation for the $\beta$ values obtained: if a firm is composed of perfectly independent subunits, a value near $\beta=-0.5$ should we expect while a value of $\beta=0$ would be expected if the growth rates of divisions of a large diversified firm are perfectly correlated. A $\beta$ value equal or close to -0.5 was obtained for small firms most likely composed of a single division, while for larger-sized firms a $\beta$ value near 0 suggest that units of larger firms have growth trends that have some correlation.

Finally, in this empirical study we find that the size distribution is best fitted by the generalized Pareto distribution. The main disadvantage of the Pareto distribution is that it fails to explain a small part of the size distribution

- its initial part that corresponds to the micro/small-sized firms. However, in Portugal and Spain, and consequently in our data, most of the firms lay on that part of the distribution. Thus, an accurate fit for the size distribution of the majority of the firms in Iberia was important to motivate the distinction highlighted here between the behavior of smaller and larger firms. As future work we plan to develop theoretical models in which firms' growth rates follow a Cauchy distribution in order to be consistent with the empirical densities found in this study.

\section{Acknowledgements}

This work has been supported by the Fundação para a Ciência e a Tecnologia (FCT) under project grant UID/MULTI/00308/2013.

\section{References}

[1] M.H.R. Stanley, L.A.N. Amaral, S.V. Buldyrev, S. Havlin, H. Leschhorn, P. Maass, M.A. Salinger, H.E. Stanley, Scaling behaviour in the growth of companies, Nature 379 (1996) 804-806.

[2] L.A.N. Amaral, S.V. Buldyrev, S. Havlin, H. Leschhorn, P. Maass, M.A. Salinger, H.E. Stanley, M.H.R. Stanley, Scaling behavior in economics: I. Empirical results for company growth, Journal de Physique I 7 (1997) 621633.

[3] L.A.N. Amaral, S.V. Buldyrev, S. Havlin, M.A. Salinger, H.E. Stanley, Power law scaling for a system of interacting units with complex internal structure, Physical Review Letters 80 (1998) 1385-1388. 
[4] Y. Lee, L.A.N. Amaral, D. Canning, M. Meyer, H.E. Stanley, Universal features in the growth dynamics of complex organizations, Physical Review Letters 81 (1998) 3275-3278.

[5] R. Axtell, D. Teitelbaum, Extreme fluctuations in US. Firm growth rates. Working paper (2004).

[6] G. Bottazzi, A. Secchi, Explaining the distribution of firm growth rates, RAND Journal of Economics 37 (2006) 235-256.

[7] R. Gibrat, Les Inégalités économiques, Recueil Sirey, Paris (1931).

[8] W.J. Xie, G.F., Gu. W.X., Zhou, On the growth of primary industry and population of China's counties, Physica A 389 (2010) 3876-3882.

[9] D. Fu, F. Pammolli, S.V. Buldyrev, M. Riccaboni, K. Matia, K. Yamasaki, H.E. Stanley, The growth of business firms: Theoretical framework and empirical evidence, PNAS 102 (2005) 18801-18806.

[10] S.V. Buldyrev, M. Riccaboni, J. Growiec, H.E. Stanley,F. Pammolli, The Growth of Business Firms: Facts and Theory, Journal of the European Economic Association 5 (2007) 574-584.

[11] M.A. Williams, B.P. Pinto, D. Park, Global evidence on the distribution of firm growth rates, Physica A 432 (2015) 102-107.

[12] M.A. Williams, G. Baek, Y. Li, L.Y. Park, W. Zhao, Global evidence on the distribution of economic profit rates, Physica A 458 (2016) 356-363.

[13] M.A. Williams, G. Baek, L.Y. Park, W. Zhao, Global evidence on the distribution of GDP growth rates, Physica A 468 (2017) 750-758.

[14] G. Bottazzi, A. Secchi, Gibrat law and diversification. Industrial and Corporate Change 15 (2006) 847-875.

[15] S. Hymer, P. Pashigian, Firm size and rate of growth, Journal of Political Economy 70 (1962) 556-569.

[16] E. Mansfield, Entry, Gibrats law, innovation and the growth of firms, American Economic Review 52 (1962) 1023-1051.

[17] A. Coad, Firm growth: a survey, Papers on Economics and Evolution 200703, Max Planck Institute of Economics, Germany (2007).

[18] Annual Report on European SMEs, European Union, 2017. https://ec.europa.eu/growth/smes

[19] World Bank national accounts data, World Bank Group. https://data.worldbank.org/

[20] R. Pascoal, M. Augusto, A. M. Monteiro, Size distribution of Portuguese firms between 2006 and 2012, Physica A 458 (2016) 342-355. 
[21] J. Steindl, Random processes and the growth of firms, London:Griffin (2004).

[22] L.A. Nunes Amaral, S.V. Buldyrev, S. Havlin, P. Maass, M.A. Salinger, H. Eugene Stanley, M.H. Stanley, Scaling behavior in economics: the problem of quantifying company growth, Physica A 244 (1997) 1-24.

[23] G. Bottazzi, G. Dosi, M. Lippi, F. Pammolli, M. Riccaboni, Innovation and corporate growth in the evolution of the drug industry, Int. J. Ind. Organiz. 19 (2001) 1161-1187.

[24] G. Bottazzi, A. Secchi, Why are distributions of firm growth rates tentshaped? Econom. Lett. 80 (3) (2003) 415420.

[25] S.V. Buldyrev, J. Growiec, F. Pammolli, M. Riccaboni, H.E. Stanley, The growth of business firms: facts and theory, J. Eur. Econ. Assoc. 5 (2007) $574-584$.

[26] S. Alfarano, M. Milakovi, Does classical competition explain the statistical features of firm growth? Econom. Lett. 101 (2008) 272-274.

[27] M. Riccaboni, J. Growiec, F. Pammolli, Innovation and Corporate Dynamics: a Theoretical Framework. Tech. Rep., University Library of Munich, Germany, 2011.

[28] W. Holzl, Persistence, survival and growth: a closer look at 20 years of high growth firms and firm dynamics in Austria, in: Workshop on High-Growth Firms, Stockholm, Sweden, 2011.

[29] E.J. Erlingsson, S. Alfarano, M. Raberto, H. Stefnsson, On the distributional properties of size, profit and growth of Icelandic firms, J. Econ. Interact. Coord. 8 (2013) 57-74.

[30] G. Bottazzi, A. Coad, N. Jacobi, A. Secchi, Corporate growth and industrial dynamics: evidence from French manufacturing, Applied Economics 43 (2011) 103-116.

[31] P.E. Hart, The Size and Growth of Firms, Economica 29 (1962) 29-39.

[32] J. Samuels, Size and the growth of firms, Review of Economic Studies 32 (1965) 105-112.

[33] A. Singh, G. Whittington, The Size and Growth of Firms, Review of Economic Studies 42 (1975) 15-26.

[34] M.S. Kumar, Growth, Acquisition Activity and Firm Size: Evidence from the United Kingdom, Journal of Industrial Economics 33 (1985) 327-338.

[35] P. Dunne, A. Hughes, Age, Size, Growth and Survival: UK companies in the 1980s, Journal of Industrial Economics 42 (1994) 115-140. 
[36] T. Yasuda, Firm Growth, Size, Age and Behavior in Japanese Manufacturing, Small Business Economics 24 (2005) 1-15.

[37] R.E. Caves, Industrial Organization and New Findings on the Turnover and Mobility of Firms, Journal of Economic Literature 36 (1998) 1947-1982. 